\title{
EFFECTS OF SHAPE ON THE WIND-INSTIGATE RESPONSE OF HIGH RISE BUILDINGS
}

\author{
M. R. Wakchaure ${ }^{1}$, Sayali Gawali ${ }^{2}$ \\ ${ }^{1}$ Professor, Civil Engineering Department, Amrutvahini College of Engineering Sangamner, Maharashtra, India \\ ${ }^{2}$ Post Graduate Student, Civil Engineering Department, Amrutvahini College of Engineering Sangamner, \\ Maharashtra, India
}

\begin{abstract}
A large number of structures that are being constructed at present tend to be wind-sensitive because of their slenderness, shapes, size, lightness and flexibility. With the ever increase in the vertical growth of urban cities, high rise buildings are being constructed in large numbers. In this study, analytical investigation of different shapes of buildings are taken as an example and various analytical approaches are performed on the building. These plans are modeled and wind loads are found out according to I.S 875(part 3)-1987 by taking gust factor and without taking gust factor. These models are compared in different aspects such as storey drift, storey displacement, storey shear, etc. for different shapes of buildings by using finite element software package ETAB's 13.1.1v. Among these results, which shape of building provide sound wind loading to the structure as well as the structural efficiency would be selected.
\end{abstract}

Key Words: Storey displacement, Storey drift, Storey shear, Gust, Wind load

\section{INTRODUCTION}

A building having height more than $15 \mathrm{~m}$ as per National building code 2005 of India is called high rise building. Due to rapid growth of population, expensive land prices, to improve aesthetic view of city and restriction in horizontal development due to less space, vertical growth is the ultimate option available. Wind is the large scale horizontal movement of free air. It plays an important role in design of tall structures because it exerts loads on buildings. The response of structures to wind depends on the characteristics of wind. As the height of building increases, there is more danger against high velocity of wind force at high level.

\subsection{Wind Effects on Structures}

Wind is randomly varying dynamic phenomenon. Wind loading is complex live load that varies both in time and space. Wind effects on structures can be classified as static and dynamic. Static wind effects causes elastic bending and twisting of structure. Wind gusts are the major dynamic effects on the on the structures which induce large dynamic motions, including oscillations. Hence, dynamic analysis is essential for tall structures.

It is important to evaluate the characteristics of fluctuating wind forces and the dynamic characteristics of the building. The wind induced building response of tall buildings can be reduced by means of aerodynamic based design and modifications that change the flow pattern around the building or break up the wind affecting the building face. The use of aerodynamic building forms is an effective method of reducing the wind loads on buildings.

\section{METHODOLOGY}

In this study, the gust response factors and the equivalent static wind loads for various along wind response components at different shape of building as per I.S 875(part3)-1987 are calculated and analysed with the help of ETAB's 13.1.1v.

Table -1: Parameters considered for the study

\begin{tabular}{|l|l|}
\hline No. of Storey & 50 \\
\hline Bottom storey height & $3 \mathrm{~m}$ \\
\hline Storey height & $3 \mathrm{~m}$ \\
\hline Soil type & Medium \\
\hline Wind zone & $\mathrm{I}$ \\
\hline Shape of buildings & $\begin{array}{l}\text { Rectangular, square, } \\
\text { circular and elliptical }\end{array}$ \\
\hline Thickness of slab & $0.125 \mathrm{~m}$ \\
\hline Beam size & $0.3 \mathrm{~m} \mathrm{x} 0.6 \mathrm{~m}$ \\
\hline Column size & $1 \mathrm{~m} \mathrm{x} 1 \mathrm{~m}$ \\
\hline Material Properties & \\
\hline Grade of concrete & $\mathrm{M} 40$ \\
\hline Grade of steel & Fe 500 \\
\hline Dead load intensities & \\
\hline FF on floors & $1.75 \mathrm{kN} / \mathrm{m}^{2}$ \\
\hline FF on roof & $2 \mathrm{kN} / \mathrm{m}^{2}$ \\
\hline Live load intensities & \\
\hline LL on floors & $2 \mathrm{kN} / \mathrm{m}^{2}$ \\
\hline LL on roof & $1 \mathrm{kN} / \mathrm{m}^{2}$ \\
\hline
\end{tabular}




\subsection{Building Models}

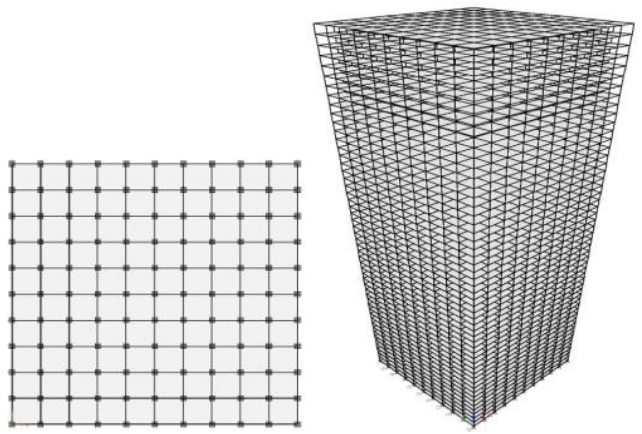

Fig-1: Plan and 3-D view of square building

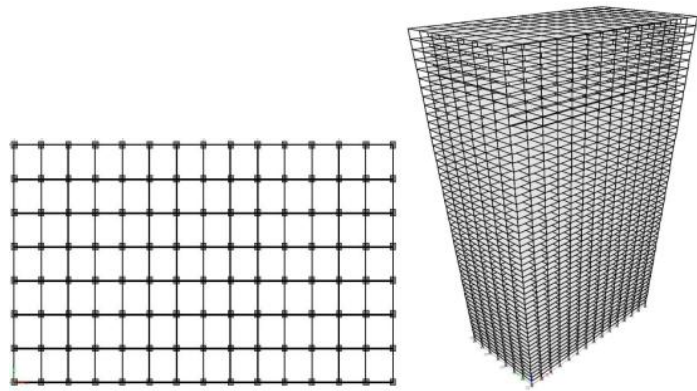

Fig-2: Plan and 3-D view of rectangular building

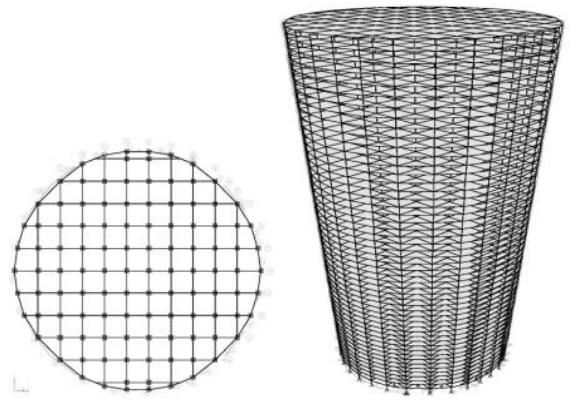

Fig-3: Plan and 3-D view of circular building
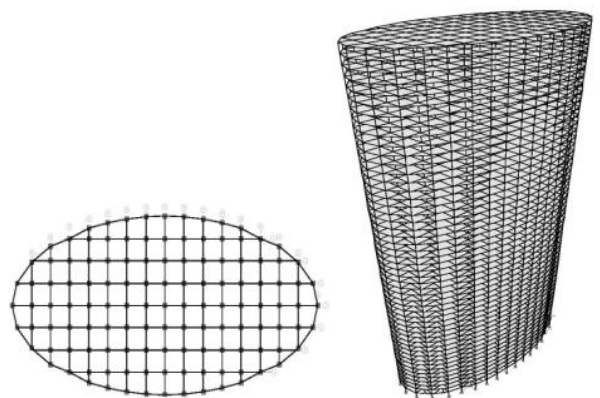

Fig-4: Plan and 3-D view of elliptical building

Table -2: Model Plan dimensions

\begin{tabular}{|l|c|c|c|c|}
\hline $\begin{array}{c}\text { Name of } \\
\text { parameter }\end{array}$ & $\begin{array}{c}\text { Square } \\
\text { shape }\end{array}$ & $\begin{array}{c}\text { Rectangul } \\
\text { ar shape }\end{array}$ & $\begin{array}{c}\text { Circular } \\
\text { shape }\end{array}$ & $\begin{array}{c}\text { Elliptical } \\
\text { shape }\end{array}$ \\
\hline $\begin{array}{l}\text { Plan dimension in } \\
\text { x-direction }\end{array}$ & $50 \mathrm{~m}$ & $70 \mathrm{~m}$ & $28.20 \mathrm{~m}$ & $38 \mathrm{~m}$ \\
\hline $\begin{array}{l}\text { Plan dimension in } \\
\text { y-direction }\end{array}$ & $50 \mathrm{~m}$ & $35.714 \mathrm{~m}$ & $28.20 \mathrm{~m}$ & $20.95 \mathrm{~m}$ \\
\hline
\end{tabular}

\subsection{Linear Analysis}

\subsubsection{Pressure coefficients}

Pressure coefficients are applicable to structural elements like walls and roofs as well as to the design of cladding. The calculation process implies the algebraic addition of $\mathrm{C}_{\mathrm{pe}}$ and $\mathrm{C}_{\mathrm{pi}}$ obtain the final wind loading. External pressure coefficient depends on wind direction, structure configuration in plan, its height versus width ratio and, characteristics of roof and its shape. Internal pressure coefficients are largely dependent on the percentage of openings in the walls and their location with reference to wind direction.

\subsubsection{Force coefficients}

The value of force coefficients apply to a building or structure as a whole, and when multiplied by the effective frontal area $\mathrm{A}$, of the building or structure and by design wind pressure, $\mathrm{p}_{\mathrm{d}}$ gives the total wind load on that particular building or structure.

\subsubsection{Parameters considered for linear analysis}

Design wind speed: The basic wind speed $\left(\mathrm{V}_{\mathrm{z}}\right)$ for any site shall be obtained from Fig.1 IS: (875(Part 3)-1987) and shall be modified to include the following effects to get design wind velocity at any height $\left(\mathrm{V}_{\mathrm{z}}\right)$ for the chosen structure:
a) Risk level
b) Terrain roughness, height and size of structure; and
c) Local topography

$\mathrm{V}_{\mathrm{z}}=\mathrm{V}_{\mathrm{b}} \cdot \mathrm{k}_{1} \cdot \mathrm{k}_{2} \cdot \mathrm{k}_{3}$

$\mathrm{V}_{\mathrm{z}}=$ hourly mean wind speed in $\mathrm{m} / \mathrm{s}$, at height $\mathrm{z}$

$\mathrm{V}_{\mathrm{b}}=$ regional basic wind speed in $\mathrm{m} / \mathrm{s}$

$\mathrm{k}_{1}=$ probability factor (risk coefficient) (Clause 5.3.1 of IS: 875(Part 3)-1987)

$\mathrm{k}_{2}=$ Terrain and height factor (Clause 5.3.2 of IS: 875 (Part 3)-1987)

$\mathrm{k}_{3}=$ topography factor (Clause 5.3.3 of IS: 875 (Part 3)1987)

Design Wind Pressure: The design wind pressure at any height above mean level shall be obtained by the Following relationship between wind pressure and wind velocity:

$\mathrm{P}_{\mathrm{z}}=0.6 \mathrm{~V}_{\mathrm{z}}{ }^{2}$

Where, $\mathrm{P}_{\mathrm{z}}=$ Design wind pressure in $\mathrm{N} / \mathrm{m} 2$ at height ' $\mathrm{z}$ ' $\mathrm{m}$ $\mathrm{V}_{\mathrm{z}}=$ design wind velocity in $\mathrm{m} / \mathrm{s}$ at height , $\mathrm{Z}^{\mathrm{ec}} \mathrm{m}$

Wind Load on Individual Members: (IS: 875 (Part 3)

$\mathrm{F}=\left(\mathrm{C}_{\mathrm{pe}}-\mathrm{C}_{\mathrm{pi}}\right) \mathrm{AP}_{\mathrm{Z}}$

Where, $\mathrm{C}_{\mathrm{pe}}=$ external pressure coefficient,

$\mathrm{C}_{\mathrm{pi}}=$ internal pressure- coefficient,

$\mathrm{A}=$ surface area of structural or cladding unit and

$\mathrm{P}_{\mathrm{z}}=$ design wind pressure.

Table -3: Parameters considered for linear analysis

\begin{tabular}{|l|c|c|c|c|}
\hline $\begin{array}{c}\text { Name of } \\
\text { parameter }\end{array}$ & $\begin{array}{c}\text { Square } \\
\text { shape }\end{array}$ & $\begin{array}{c}\text { Rectangular } \\
\text { shape }\end{array}$ & $\begin{array}{c}\text { Circular } \\
\text { shape }\end{array}$ & $\begin{array}{c}\text { Elliptical } \\
\text { shape }\end{array}$ \\
\hline$\left(\mathrm{k}_{1}\right)$ & 1 & 1 & 1 & 1 \\
\hline$\left(\mathrm{k}_{3}\right)$ & 1 & 1 & 1 & 1 \\
\hline Terrain category & II & II & II & II \\
\hline Class of structure & B & C & C & C \\
\hline
\end{tabular}


Table -4: Linear Wind loads (KN)

\begin{tabular}{|c|c|c|c|c|c|}
\hline \multirow{2}{*}{$\begin{array}{l}\text { Floo } \\
\text { r no. }\end{array}$} & \multirow{2}{*}{$\begin{array}{c}\text { Squar } \\
\mathrm{e}\end{array}$} & \multicolumn{2}{|c|}{ Rectangular } & \multirow[b]{2}{*}{ Circular } & \multirow[b]{2}{*}{ Elliptical } \\
\hline & & $\mathrm{X}$ & Y & & \\
\hline 1 & 131.78 & 166.15 & 84.77 & 105.17 & 32.78 \\
\hline 5 & 142.76 & 180.75 & 92.22 & 114.41 & 35.66 \\
\hline 10 & 166.03 & 207.77 & 106.01 & 131.52 & 41.00 \\
\hline 15 & 177.54 & 226.14 & 115.38 & 143.15 & 44.62 \\
\hline 20 & 185.91 & 238.39 & 121.63 & 150.91 & 47.04 \\
\hline 25 & 192.68 & 247.47 & 126.26 & 156.65 & 48.83 \\
\hline 30 & 199.57 & 256.71 & 130.97 & 162.50 & 50.65 \\
\hline 35 & 205.23 & 263.41 & 134.40 & 166.74 & 51.98 \\
\hline 40 & 208.27 & 269.98 & 137.74 & 170.90 & 53.27 \\
\hline 45 & 211.32 & 275.70 & 140.66 & 174.52 & 54.40 \\
\hline 50 & 214.40 & 281.25 & 143.50 & 178.04 & 55.50 \\
\hline
\end{tabular}

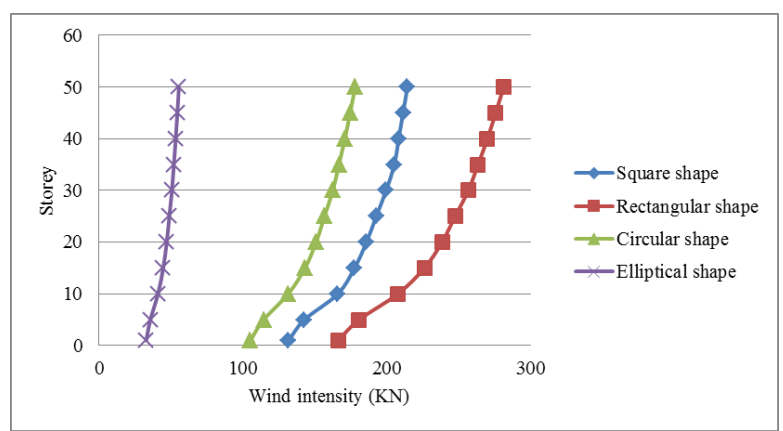

Chart -1: Wind intensity in X-direction

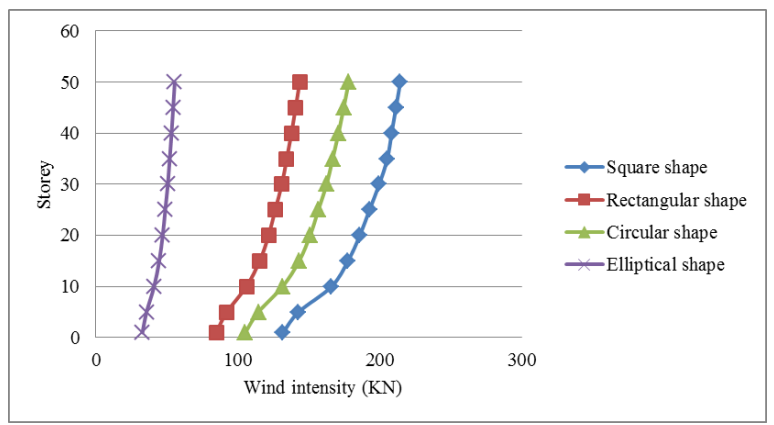

Chart -2: Wind intensity in Y-direction

From linear analysis, it was seen from table- 4 that with the increase in the height of building the wind intensities were also increasing and wind intensities were decreasing with the variation of shapes from square to elliptical. The variation of wind intensity is shown in chart 1 and chart 2 .

\subsection{Gust Factor Method}

A gust factor, defined as the ratio between a peak wind gust and mean wind speed over a period of time, can be used along with other statistics to examine the structure of the wind.

Constants and parameters used for gust factor analysis are:

i. $\quad \mathrm{T}=$ Time period (pg.48, IS 875(part-3)-1987)

ii. $\quad \mathrm{C}_{\mathrm{f}}=$ Force coefficient for clad building (fig. 4 of IS 875(part-3)-1987)

iii. $\quad g_{\mathrm{f}}=$ Peak Factor and Roughness Factor (fig. 8 of IS 875(part-3)-1987)

iv. $\quad \mathrm{B}=$ Background factor (fig. 9 of IS 875(part-3)1987)

v. $\quad S=$ Size reduction factor (fig. 10 of IS 875(part-3)1987)

vi. $\varnothing=$ Constant

vii. $\quad \mathrm{E}=$ Gust energy factor (fig. 11 of IS 875(part-3)1987)

viii. $\quad \beta=$ (pg.52, IS 875(part-3)-1987)

ix. $\mathrm{G}=$ Gust factor

$G=1+g f r \sqrt{\left[B(1+\phi)^{2}+\frac{S E}{\beta}\right]}$

x. $\quad \mathrm{F}_{\mathrm{x}}=$ Along wind load on the structure on a strip area at any height

$F x=C f \cdot A e \cdot P z \cdot G$

Table -5: Parameters considered for gust factor analysis

\begin{tabular}{|c|c|c|c|c|}
\hline $\begin{array}{c}\text { Name of } \\
\text { parameter }\end{array}$ & $\begin{array}{c}\text { Square } \\
\text { model }\end{array}$ & $\begin{array}{c}\text { Rectangular } \\
\text { model }\end{array}$ & $\begin{array}{c}\text { Circular } \\
\text { model }\end{array}$ & $\begin{array}{c}\text { Elliptical } \\
\text { model }\end{array}$ \\
\hline $\mathrm{T}_{\mathrm{x}}$ & 1.9 & 1.61 & 2.54 & 2.18 \\
\hline $\mathrm{T}_{\mathrm{y}}$ & 1.9 & 2.25 & 2.54 & 2.94 \\
\hline $\mathrm{C}_{\mathrm{f}}$ & 1.25 & 1.1 & 0.7 & 0.2 \\
\hline $\mathrm{g}_{\mathrm{f}}$ & 0.85 & 0.85 & 0.85 & 0.85 \\
\hline $\mathrm{B}$ & 0.85 & 0.85 & 0.85 & 0.85 \\
\hline$\varnothing$ & 0 & 0 & 0 & 0 \\
\hline$\beta$ & 0.016 & 0.016 & 0.016 & 0.016 \\
\hline
\end{tabular}

Table -6: Wind loads with gust factor (KN)

\begin{tabular}{|c|c|c|c|c|c|c|}
\hline \multirow{2}{*}{$\begin{array}{c}\text { Floor } \\
\text { no. }\end{array}$} & Square & \multicolumn{2}{|c|}{ Rectangular } & Circular & \multicolumn{2}{|c|}{ Elliptical } \\
\cline { 3 - 4 } \cline { 6 - 7 } & & $\mathrm{X}$ & $\mathrm{Y}$ & & $\mathrm{X}$ & $\mathrm{Y}$ \\
\hline 1 & 96.46 & 116.57 & 67.26 & 94.77 & 30.17 & 29.22 \\
\hline 5 & 111.76 & 134.82 & 78.52 & 109.81 & 35.17 & 33.85 \\
\hline 10 & 136.45 & 162.67 & 95.85 & 132.90 & 42.91 & 40.80 \\
\hline 15 & 153.23 & 181.94 & 107.96 & 149.22 & 48.41 & 45.84 \\
\hline 20 & 165.27 & 194.96 & 116.17 & 160.49 & 52.06 & 49.17 \\
\hline 25 & 175.29 & 204.68 & 122.30 & 168.74 & 54.74 & 51.67 \\
\hline 30 & 183.98 & 214.61 & 128.60 & 177.10 & 57.98 & 54.23 \\
\hline 35 & 191.70 & 223.33 & 134.15 & 184.53 & 60.85 & 56.44 \\
\hline 40 & 197.03 & 229.23 & 137.91 & 189.55 & 62.69 & 57.96 \\
\hline 45 & 203.04 & 235.21 & 141.72 & 194.60 & 64.47 & 59.52 \\
\hline 50 & 209.79 & 241.27 & 145.57 & 200.41 & 66.31 & 61.10 \\
\hline
\end{tabular}

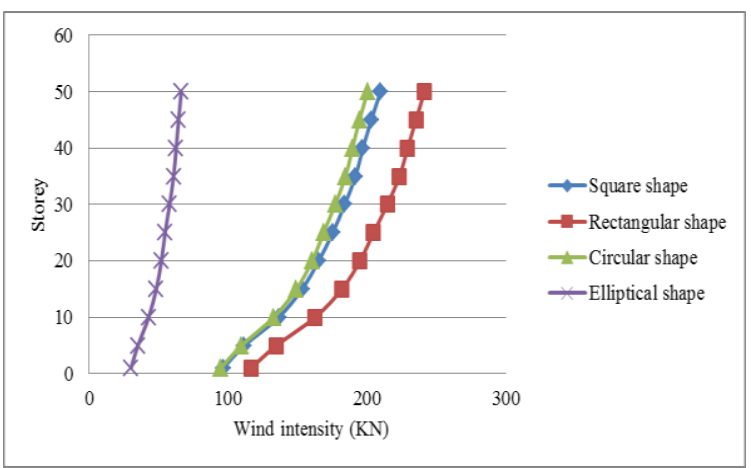

Chart -3: Wind intensity in X-direction 


\section{RESULTS AND DISCUSSION}

In this section, behavior of different buildings when subjected to wind load have been discussed.

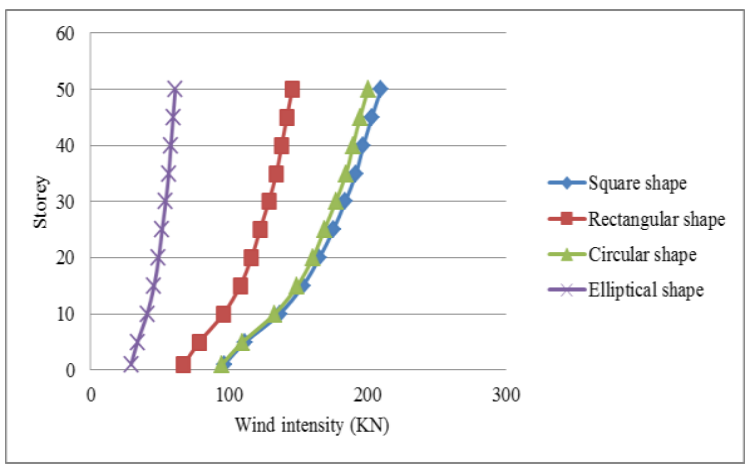

Chart -4: Wind intensity in Y-direction

\subsection{Wind Intensity}

It is the pressure exerted by the wind on the structure. The wind intensity for square, rectangular, circular and elliptical shape building are compared in table 7 and it is graphically represented in chart $5,6,7$ and 8 .

Table -7: Comparison of wind loads without gust factor (X-direction) $(\mathrm{kN})$

\begin{tabular}{|c|c|c|c|c|c|c|c|c|}
\hline \multirow{2}{*}{$\begin{array}{c}\text { Storey } \\
\text { No. }\end{array}$} & Height & Square & \multicolumn{2}{|c|}{ Rectangular } & \multicolumn{2}{|c|}{ Circular } & \multicolumn{2}{|c|}{ Elliptical } \\
\cline { 3 - 9 } & & $\begin{array}{c}\text { Wind } \\
\text { intensity } \\
(\mathrm{kN})\end{array}$ & $\begin{array}{c}\text { Wind } \\
\text { intensity } \\
(\mathrm{kN})\end{array}$ & $\begin{array}{c}\% \\
\text { Decrease }\end{array}$ & $\begin{array}{c}\text { Wind } \\
\text { intensity } \\
(\mathrm{kN})\end{array}$ & $\begin{array}{c}\% \\
\text { Decrease }\end{array}$ & $\begin{array}{c}\text { Wind } \\
\text { intensity } \\
(\mathrm{kN})\end{array}$ & $\begin{array}{c}\% \\
\text { Decrease }\end{array}$ \\
\hline 0 & 0 & 131.780 & 166.147 & -26.079 & 105.172 & 20.191 & 32.784 & 75.123 \\
\hline 10 & 30 & 166.029 & 207.775 & -25.144 & 131.523 & 20.783 & 40.997 & 75.307 \\
\hline 20 & 60 & 185.911 & 238.395 & -28.231 & 150.906 & 18.829 & 47.039 & 74.698 \\
\hline 30 & 90 & 199.569 & 256.710 & -28.632 & 162.499 & 18.575 & 50.653 & 74.619 \\
\hline 40 & 120 & 208.267 & 269.979 & -29.631 & 170.899 & 17.942 & 53.271 & 74.422 \\
\hline 50 & 150 & 214.397 & 281.253 & -31.183 & 178.036 & 16.960 & 55.496 & 74.115 \\
\hline
\end{tabular}

Table -8: Comparison of wind loads without gust factor (Y-direction) $(\mathrm{kN})$

\begin{tabular}{|c|c|c|c|c|c|c|c|c|}
\hline \multirow{2}{*}{$\begin{array}{c}\text { Storey } \\
\text { No. }\end{array}$} & \multirow{2}{*}{$\begin{array}{l}\text { Height } \\
\text { m }\end{array}$} & Square & \multicolumn{2}{|c|}{ Rectangular } & \multicolumn{2}{|c|}{ Circular } & \multicolumn{2}{|c|}{ Elliptical } \\
\hline & & $\begin{array}{c}\text { Wind } \\
\text { intensity } \\
(\mathrm{kN})\end{array}$ & $\begin{array}{c}\text { Wind } \\
\text { intensity } \\
(\mathrm{kN})\end{array}$ & $\begin{array}{c}\% \\
\text { Decrease }\end{array}$ & $\begin{array}{c}\text { Wind } \\
\text { intensity } \\
(\mathrm{kN})\end{array}$ & $\begin{array}{c}\% \\
\text { Decrease }\end{array}$ & $\begin{array}{c}\text { Wind } \\
\text { intensity } \\
(\mathrm{kN})\end{array}$ & $\begin{array}{c}\% \\
\text { Decrease }\end{array}$ \\
\hline 0 & 0 & 131.780 & 84.769 & 35.674 & 105.172 & 20.191 & 32.784 & 75.123 \\
\hline 10 & 30 & 166.029 & 106.008 & 36.151 & 131.523 & 20.783 & 40.997 & 75.307 \\
\hline 20 & 60 & 185.911 & 121.630 & 34.576 & 150.906 & 18.829 & 47.039 & 74.698 \\
\hline 30 & 90 & 199.569 & 130.974 & 34.371 & 162.499 & 18.575 & 50.653 & 74.619 \\
\hline 40 & 120 & 208.267 & 137.744 & 33.862 & 170.899 & 17.942 & 53.271 & 74.422 \\
\hline 50 & 150 & 214.397 & 143.496 & 33.070 & 178.036 & 16.960 & 55.496 & 74.115 \\
\hline
\end{tabular}

Table -9: Comparison of wind loads with gust factor (X-direction) (kN)

\begin{tabular}{|c|c|c|c|c|c|c|c|c|}
\hline \multirow{2}{*}{$\begin{array}{l}\text { Storey } \\
\text { No. }\end{array}$} & \multirow{2}{*}{$\begin{array}{l}\text { Height } \\
\text { m }\end{array}$} & Square & \multicolumn{2}{|c|}{ Rectangular } & \multicolumn{2}{|c|}{ Circular } & \multicolumn{2}{|c|}{ Elliptical } \\
\hline & & $\begin{array}{c}\text { Wind } \\
\text { intensity } \\
(\mathrm{kN})\end{array}$ & $\begin{array}{c}\text { Wind } \\
\text { intensity } \\
(\mathrm{kN})\end{array}$ & $\begin{array}{c}\% \\
\text { Decrease }\end{array}$ & $\begin{array}{l}\text { Wind } \\
\text { intensity } \\
(\mathrm{kN})\end{array}$ & $\begin{array}{c}\% \\
\text { Decrease }\end{array}$ & $\begin{array}{c}\text { Wind } \\
\text { intensity } \\
(\mathrm{kN})\end{array}$ & $\begin{array}{c}\% \\
\text { Decrease }\end{array}$ \\
\hline 0 & 0 & 96.457 & 116.566 & -20.847 & 94.773 & 1.746 & 30.172 & 68.719 \\
\hline 10 & 30 & 136.446 & 162.674 & -19.223 & 132.902 & 2.597 & 42.915 & 68.548 \\
\hline 20 & 60 & 165.272 & 194.964 & -17.965 & 160.494 & 2.891 & 52.061 & 68.500 \\
\hline 30 & 90 & 183.979 & 214.612 & -16.651 & 177.101 & 3.738 & 57.981 & 68.485 \\
\hline 40 & 120 & 197.028 & 229.230 & -16.344 & 189.554 & 3.793 & 62.695 & 68.180 \\
\hline 50 & 150 & 209.786 & 241.265 & -15.006 & 200.406 & 4.471 & 66.310 & 68.392 \\
\hline
\end{tabular}


Table -10: Comparison of wind loads with gust factor (Y-direction) $(\mathrm{kN})$

\begin{tabular}{|c|c|c|c|c|c|c|c|c|}
\hline \multirow{2}{*}{$\begin{array}{c}\text { Storey } \\
\text { No. }\end{array}$} & \multirow{2}{*}{$\begin{array}{l}\text { Height } \\
\mathrm{m}\end{array}$} & \multirow{2}{*}{$\begin{array}{c}\text { Square } \\
\text { Wind } \\
\text { intensity } \\
(\mathrm{kN})\end{array}$} & \multicolumn{2}{|c|}{ Rectangular } & \multicolumn{2}{|c|}{ Circular } & \multicolumn{2}{|c|}{ Elliptical } \\
\hline & & & $\begin{array}{c}\text { Wind } \\
\text { intensity } \\
(\mathrm{kN})\end{array}$ & $\begin{array}{c}\% \\
\text { Decrease }\end{array}$ & $\begin{array}{c}\text { Wind } \\
\text { intensity } \\
(\mathrm{kN})\end{array}$ & $\begin{array}{c}\% \\
\text { Decrease }\end{array}$ & $\begin{array}{l}\text { Wind } \\
\text { intensity } \\
(\mathrm{kN})\end{array}$ & $\begin{array}{c}\% \\
\text { Decrease }\end{array}$ \\
\hline 0 & 0 & 96.457 & 67.265 & 30.265 & 94.773 & 1.746 & 29.222 & 69.704 \\
\hline 10 & 30 & 136.446 & 95.846 & 29.755 & 132.902 & 2.597 & 40.803 & 70.096 \\
\hline 20 & 60 & 165.272 & 116.165 & 29.713 & 160.494 & 2.891 & 49.174 & 70.247 \\
\hline 30 & 90 & 183.979 & 128.604 & 30.099 & 177.101 & 3.738 & 54.235 & 70.521 \\
\hline 40 & 120 & 197.028 & 137.912 & 30.004 & 189.554 & 3.793 & 57.959 & 70.583 \\
\hline 50 & 150 & 209.786 & 145.574 & 30.608 & 200.406 & 4.471 & 61.101 & 70.875 \\
\hline
\end{tabular}

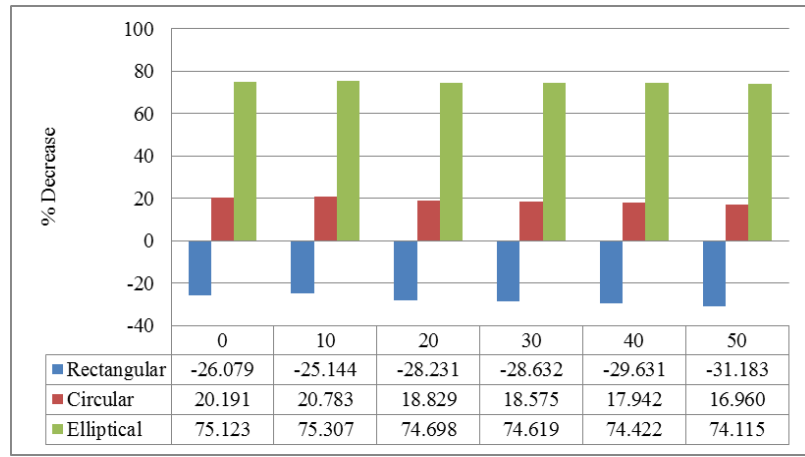

Chart -5: Wind intensity in X-direction (without gust)

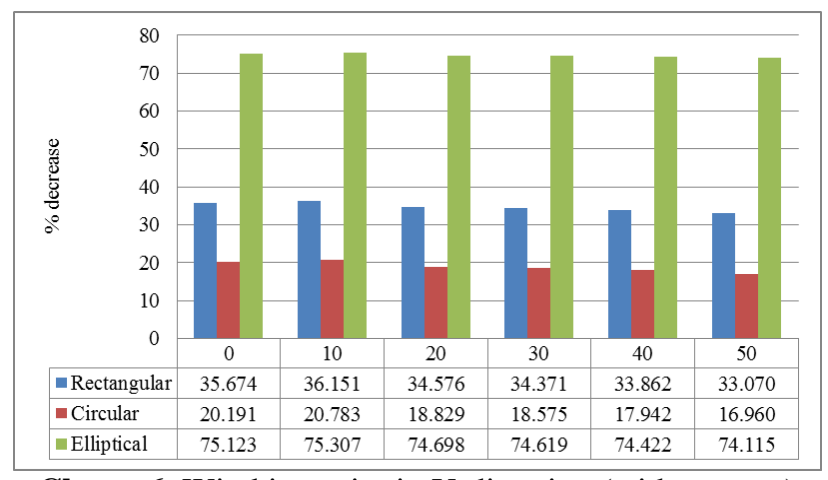

Chart -6: Wind intensity in Y-direction (without gust)

\subsection{Storey Displacement}

It is displacement caused by the lateral force on the each storey level of structure. The storey displacement for square,

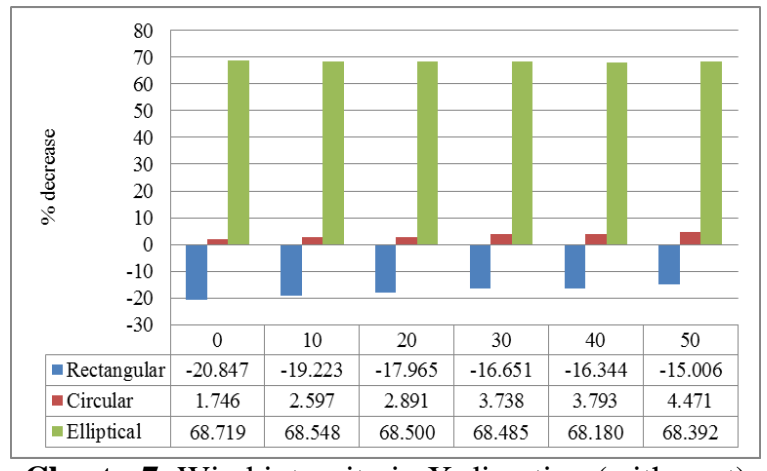

Chart -7: Wind intensity in X-direction (with gust)

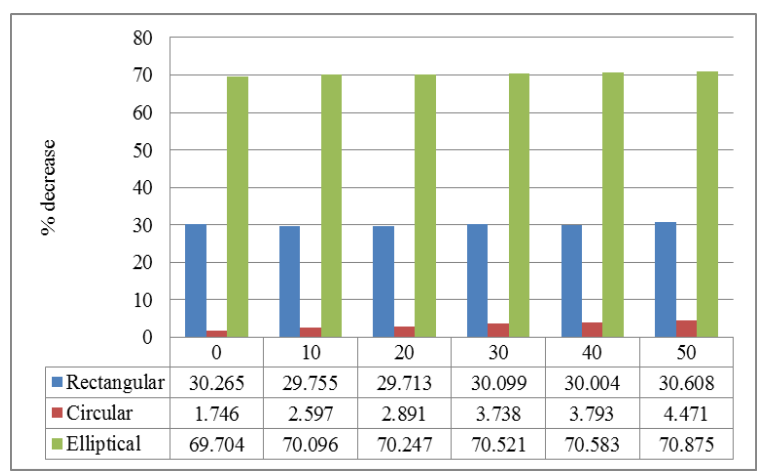

Chart -8: Wind intensity in Y-direction (with gust)

Table -11: Comparison of storey displacement without gust factor (X-direction) (mm)

\begin{tabular}{|c|c|c|c|c|c|c|c|c|}
\hline \multirow{2}{*}{$\begin{array}{l}\text { Storey } \\
\text { No. }\end{array}$} & \multirow{2}{*}{$\begin{array}{l}\text { Height } \\
\text { m }\end{array}$} & Square & \multicolumn{2}{|c|}{ Rectangular } & \multicolumn{2}{|c|}{ Circular } & \multicolumn{2}{|c|}{ Elliptical } \\
\hline & & $\begin{array}{c}\text { Storey } \\
\text { displacement } \\
(\mathrm{mm})\end{array}$ & $\begin{array}{c}\text { Storey } \\
\text { displacement } \\
(\mathrm{mm})\end{array}$ & $\begin{array}{c}\% \\
\text { Decrease }\end{array}$ & $\begin{array}{c}\text { Storey } \\
\text { displacement } \\
(\mathrm{mm})\end{array}$ & $\begin{array}{c}\% \\
\text { Decrease }\end{array}$ & $\begin{array}{c}\text { Storey } \\
\text { displacement } \\
(\mathrm{mm})\end{array}$ & $\begin{array}{c}\% \\
\text { Decrease }\end{array}$ \\
\hline 0 & 0 & 0.8 & 0.5 & 37.500 & 0.7 & 12.500 & 0.2 & 75.000 \\
\hline 10 & 30 & 27.6 & 17.7 & 35.870 & 24 & 13.043 & 6.5 & 76.449 \\
\hline 20 & 60 & 52.7 & 33.7 & 36.053 & 46.1 & 12.524 & 12.4 & 76.471 \\
\hline 30 & 90 & 72.5 & 46 & 36.552 & 63.7 & 12.138 & 17 & 76.552 \\
\hline 40 & 120 & 85.9 & 54.2 & 36.903 & 75.7 & 11.874 & 20.1 & 76.601 \\
\hline 50 & 150 & 92.9 & 58.3 & 37.244 & 82 & 11.733 & 21.6 & 76.749 \\
\hline
\end{tabular}


Table -12: Comparison of storey displacement without gust factor (Y-direction) (mm)

\begin{tabular}{|c|c|c|c|c|c|c|c|c|}
\hline \multirow{2}{*}{$\begin{array}{l}\text { Storey } \\
\text { No. }\end{array}$} & \multirow{2}{*}{$\begin{array}{l}\text { Height } \\
\text { m }\end{array}$} & Square & \multicolumn{2}{|c|}{ Rectangular } & \multicolumn{2}{|c|}{ Circular } & \multicolumn{2}{|c|}{ Elliptical } \\
\hline & & $\begin{array}{c}\text { Storey } \\
\text { displacement } \\
(\mathrm{mm})\end{array}$ & $\begin{array}{c}\text { Storey } \\
\text { displacement } \\
(\mathrm{mm})\end{array}$ & $\begin{array}{c}\% \\
\text { Decrease }\end{array}$ & $\begin{array}{c}\text { Storey } \\
\text { displacement } \\
(\mathrm{mm})\end{array}$ & $\begin{array}{c}\% \\
\text { Decrease }\end{array}$ & $\begin{array}{c}\text { Storey } \\
\text { displacement } \\
(\mathrm{mm})\end{array}$ & $\begin{array}{c}\% \\
\text { Decrease }\end{array}$ \\
\hline 0 & 0 & 0.8 & 1.1 & -37.500 & 0.8 & 0.000 & 0.2 & 75.000 \\
\hline 10 & 30 & 27.7 & 38.2 & -37.906 & 26 & 6.137 & 7 & 74.729 \\
\hline 20 & 60 & 53.1 & 74.1 & -39.548 & 50.3 & 5.273 & 14 & 73.635 \\
\hline 30 & 90 & 73.1 & 102.8 & -40.629 & 69.3 & 5.198 & 19.7 & 73.051 \\
\hline 40 & 120 & 86.9 & 123.1 & -41.657 & 82.3 & 5.293 & 24 & 72.382 \\
\hline 50 & 150 & 94.2 & 134.4 & -42.675 & 89.1 & 5.414 & 26.6 & 71.762 \\
\hline
\end{tabular}

Table -13: Comparison of storey displacement with gust factor (X-direction) (mm)

\begin{tabular}{|c|c|c|c|c|c|c|c|c|}
\hline \multirow[b]{2}{*}{$\begin{array}{c}\text { Storey } \\
\text { No. }\end{array}$} & \multirow{2}{*}{$\begin{array}{l}\text { Height } \\
\text { m }\end{array}$} & Square & \multicolumn{2}{|c|}{ Rectangular } & \multicolumn{2}{|c|}{ Circular } & \multicolumn{2}{|c|}{ Elliptical } \\
\hline & & $\begin{array}{c}\text { Storey } \\
\text { displacement } \\
(\mathrm{mm})\end{array}$ & $\begin{array}{c}\text { Storey } \\
\text { displacement } \\
(\mathrm{mm})\end{array}$ & $\begin{array}{c}\% \\
\text { Decrease }\end{array}$ & $\begin{array}{c}\text { Storey } \\
\text { displacement } \\
(\mathrm{mm})\end{array}$ & $\begin{array}{c}\% \\
\text { Decrease }\end{array}$ & $\begin{array}{c}\text { Storey } \\
\text { displacement } \\
(\mathrm{mm})\end{array}$ & $\begin{array}{c}\% \\
\text { Decrease }\end{array}$ \\
\hline 0 & 0 & 0.7 & 0.6 & 14.286 & 0.8 & -14.286 & 0.2 & 71.429 \\
\hline 10 & 30 & 25.2 & 21.7 & 13.889 & 26 & -3.175 & 7.4 & 70.635 \\
\hline 20 & 60 & 48.7 & 37.6 & 22.793 & 50.4 & -3.491 & 14.2 & 70.842 \\
\hline 30 & 90 & 67.5 & 49.9 & 26.074 & 69.7 & -3.259 & 19.5 & 71.111 \\
\hline 40 & 120 & 80.5 & 58.1 & 27.826 & 83 & -3.106 & 23.1 & 71.304 \\
\hline 50 & 150 & 87.4 & 62.1 & 28.947 & 90.1 & -3.089 & 24.9 & 71.510 \\
\hline
\end{tabular}

Table -14: Comparison of storey displacement with gust factor (Y-direction) (mm)

\begin{tabular}{|c|c|c|c|c|c|c|c|c|}
\hline \multirow[b]{2}{*}{$\begin{array}{c}\text { Storey } \\
\text { No. }\end{array}$} & \multirow[b]{2}{*}{$\begin{array}{l}\text { Height } \\
\text { m }\end{array}$} & \multirow{2}{*}{$\begin{array}{c}\text { Square } \\
\text { Storey } \\
\text { displacement } \\
(\mathrm{mm})\end{array}$} & \multicolumn{2}{|c|}{ Rectangular } & \multicolumn{2}{|c|}{ Circular } & \multicolumn{2}{|c|}{ Elliptical } \\
\hline & & & $\begin{array}{c}\text { Storey } \\
\text { displacement } \\
(\mathrm{mm})\end{array}$ & $\begin{array}{c}\% \\
\text { Decrease }\end{array}$ & $\begin{array}{c}\text { Storey } \\
\text { displacement } \\
(\mathrm{mm})\end{array}$ & $\begin{array}{c}\% \\
\text { Decrease }\end{array}$ & $\begin{array}{c}\text { Storey } \\
\text { displacement } \\
(\mathrm{mm})\end{array}$ & $\begin{array}{c}\% \\
\text { Decrease }\end{array}$ \\
\hline 0 & 0 & 0.7 & 1.2 & -71.429 & 0.8 & -14.286 & 0.2 & 71.429 \\
\hline 10 & 30 & 25.7 & 39.8 & -54.864 & 28.3 & -10.117 & 7.5 & 70.817 \\
\hline 20 & 60 & 50.6 & 70 & -38.340 & 55 & -8.696 & 14.9 & 70.553 \\
\hline 30 & 90 & 71.2 & 94.5 & -32.725 & 76 & -6.742 & 21.1 & 70.365 \\
\hline 40 & 120 & 86.3 & 111.8 & -29.548 & 90.4 & -4.751 & 25.7 & 70.220 \\
\hline 50 & 150 & 95.6 & 121.5 & -27.092 & 97.9 & -2.406 & 28.5 & 70.188 \\
\hline
\end{tabular}

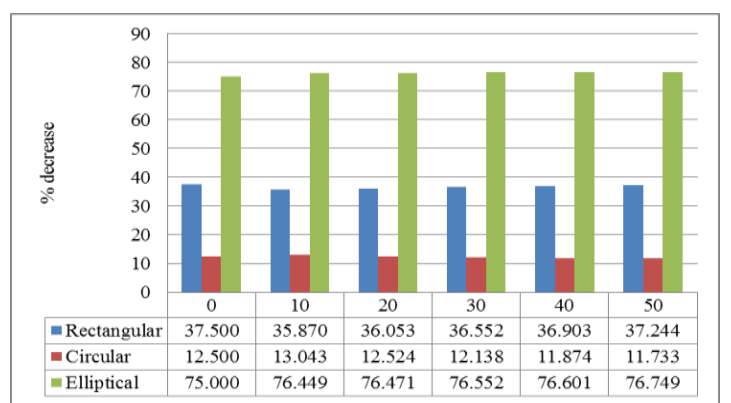

Chart -9: Storey displacement in X-direction (wog)

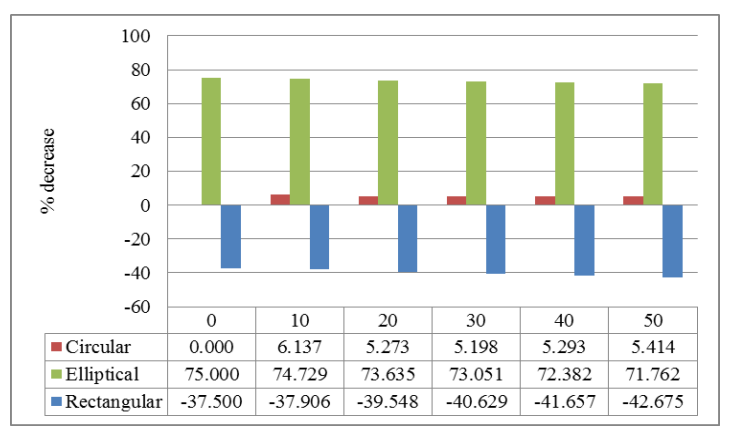

Chart -10: Storey displacement in Y-direction (wog)

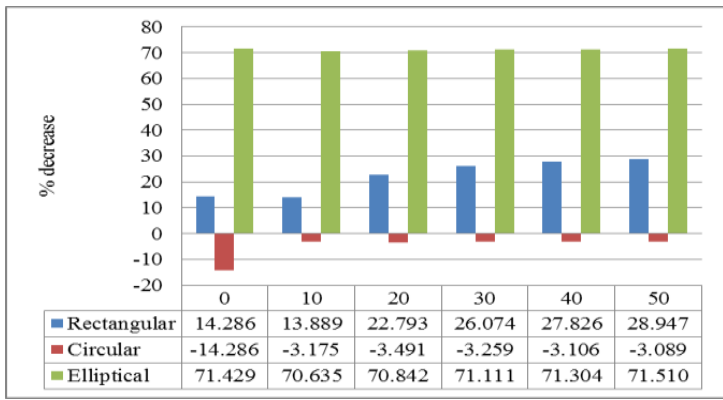

Chart -11: Storey displacement in X-direction (wg)

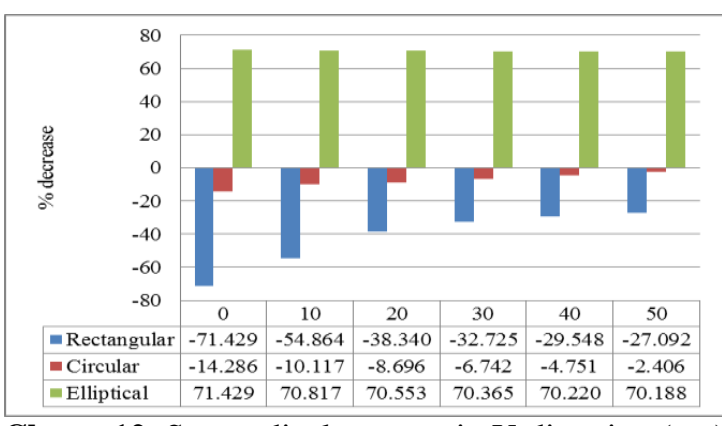

Chart -12: Storey displacement in Y-direction (wg) 


\subsection{Storey Drift}

It is the displacement of one level relative of the other level above or below. The storey drift for square, rectangular, circular and elliptical shape building are compared and it is graphically represented in chart $13,14,15,16$.

Table -15: Comparison of storey drift without gust factor (X-direction)

\begin{tabular}{|c|c|c|c|c|c|c|c|c|}
\hline \multirow{2}{*}{$\begin{array}{c}\text { Storey } \\
\text { No. }\end{array}$} & \multirow{2}{*}{$\begin{array}{c}\text { Height } \\
\mathrm{m}\end{array}$} & \begin{tabular}{c} 
Square \\
\cline { 3 - 9 }
\end{tabular} & \multicolumn{2}{|c|}{ Rectangular } & \multicolumn{2}{|c|}{ Circular } & \multicolumn{2}{|c|}{ Elliptical } \\
\hline 0 & 0 & 0.271 & 0.177 & 34.686 & 0.239 & 11.808 & 0.068 & 74.908 \\
\hline 10 & 30 & 0.924 & 0.588 & 36.364 & 0.806 & 12.771 & 0.213 & 76.948 \\
\hline 20 & 60 & 0.763 & 0.479 & 37.221 & 0.673 & 11.796 & 0.173 & 77.326 \\
\hline 30 & 90 & 0.567 & 0.349 & 38.448 & 0.503 & 11.287 & 0.125 & 77.954 \\
\hline 40 & 120 & 0.347 & 0.206 & 40.634 & 0.312 & 10.086 & 0.074 & 78.674 \\
\hline 50 & 150 & 0.2 & 0.136 & 32.000 & 0.191 & 4.500 & 0.083 & 58.500 \\
\hline
\end{tabular}

Table -16: Comparison of storey drift without gust factor (Y-direction)

\begin{tabular}{|c|c|c|c|c|c|c|c|c|}
\hline \multirow{2}{*}{$\begin{array}{c}\text { Storey } \\
\text { No. }\end{array}$} & \multirow{2}{*}{$\begin{array}{l}\text { Height } \\
\text { m }\end{array}$} & Square & \multicolumn{2}{|c|}{ Rectangular } & \multicolumn{2}{|c|}{ Circular } & \multicolumn{2}{|c|}{ Elliptical } \\
\hline & & $\begin{array}{c}\text { Storey } \\
\text { drift }\end{array}$ & Storey drift & $\begin{array}{c}\% \\
\text { Decrease }\end{array}$ & $\begin{array}{l}\text { Storey } \\
\text { drift }\end{array}$ & $\begin{array}{c}\% \\
\text { Decrease }\end{array}$ & $\begin{array}{l}\text { Storey } \\
\text { drift }\end{array}$ & $\begin{array}{c}\% \\
\text { Decrease }\end{array}$ \\
\hline 0 & 0 & 0.271 & 0.366 & -35.055 & 0.256 & 5.535 & 0.005 & 98.155 \\
\hline 10 & 30 & 0.918 & 1.3 & -41.612 & 0.883 & 3.813 & 0.002 & 99.782 \\
\hline 20 & 60 & 0.751 & 1.099 & -46.338 & 0.736 & 1.997 & 0.003 & 99.601 \\
\hline 30 & 90 & 0.551 & 0.838 & -52.087 & 0.547 & 0.726 & 0.003 & 99.456 \\
\hline 40 & 120 & 0.329 & 0.539 & -63.830 & 0.335 & -1.824 & 0.004 & 98.784 \\
\hline 50 & 150 & 0.179 & 0.297 & -65.922 & 0.205 & -14.525 & 0.061 & 65.922 \\
\hline
\end{tabular}

Table -17: Comparison of storey drift with gust factor (X-direction)

\begin{tabular}{|c|c|c|c|c|c|c|c|c|}
\hline \multirow{2}{*}{$\begin{array}{l}\text { Storey } \\
\text { No. }\end{array}$} & \multirow{2}{*}{$\begin{array}{l}\text { Height } \\
\text { m }\end{array}$} & Square & \multicolumn{2}{|c|}{ Rectangular } & \multicolumn{2}{|c|}{ Circular } & \multicolumn{2}{|c|}{ Elliptical } \\
\hline & & $\begin{array}{c}\text { Storey } \\
\text { drift } \\
(\mathrm{mm})\end{array}$ & $\begin{array}{c}\text { Storey } \\
\text { drift } \\
(\mathrm{mm})\end{array}$ & $\begin{array}{c}\% \\
\text { Decrease }\end{array}$ & $\begin{array}{c}\text { Storey } \\
\text { drift } \\
(\mathrm{mm})\end{array}$ & $\begin{array}{c}\% \\
\text { Decrease }\end{array}$ & $\begin{array}{c}\text { Storey } \\
\text { drift } \\
(\mathrm{mm})\end{array}$ & $\begin{array}{c}\% \\
\text { Decrease }\end{array}$ \\
\hline 0 & 0 & 0.246 & 0.173 & 29.675 & 0.258 & -4.878 & 0.076 & 69.106 \\
\hline 10 & 30 & 0.849 & 0.583 & 31.331 & 0.885 & -4.240 & 0.243 & 71.378 \\
\hline 20 & 60 & 0.71 & 0.482 & 32.113 & 0.741 & -4.366 & 0.2 & 71.831 \\
\hline 30 & 90 & 0.529 & 0.349 & 34.026 & 0.558 & -5.482 & 0.147 & 72.212 \\
\hline 40 & 120 & 0.323 & 0.207 & 35.913 & 0.348 & -7.740 & 0.087 & 73.065 \\
\hline 50 & 150 & 0.186 & 0.137 & 26.344 & 0.206 & -10.753 & 0.088 & 52.688 \\
\hline
\end{tabular}

Table -18: Comparison of storey drift with gust factor (Y-direction)

\begin{tabular}{|c|c|c|c|c|c|c|c|c|}
\hline \multirow{2}{*}{$\begin{array}{c}\text { Storey } \\
\text { No. }\end{array}$} & Height & Square & \multicolumn{2}{|c|}{ Rectangular } & \multicolumn{2}{|c|}{ Circular } & \multicolumn{2}{c|}{ Elliptical } \\
\cline { 3 - 9 } & & $\begin{array}{c}\text { Storey } \\
\text { drift } \\
(\mathrm{mm})\end{array}$ & $\begin{array}{c}\text { Storey drift } \\
(\mathrm{mm})\end{array}$ & $\begin{array}{c}\% \\
\text { Decrease }\end{array}$ & $\begin{array}{c}\text { Storey } \\
\text { drift } \\
(\mathrm{mm})\end{array}$ & $\begin{array}{c}\text { Storey } \\
\text { drift } \\
(\mathrm{mm})\end{array}$ & $\begin{array}{c}\% \\
\text { Decrease }\end{array}$ \\
\hline 0 & 0 & 0.244 & 0.302 & -23.770 & 0.276 & -13.115 & 0.074 & 69.672 \\
\hline 10 & 30 & 0.818 & 1.088 & -33.007 & 0.969 & -18.460 & 0.26 & 68.215 \\
\hline 20 & 60 & 0.657 & 0.929 & -41.400 & 0.81 & -23.288 & 0.232 & 64.688 \\
\hline 30 & 90 & 0.462 & 0.714 & -54.545 & 0.606 & -31.169 & 0.184 & 60.173 \\
\hline 40 & 120 & 0.247 & 0.459 & -85.830 & 0.373 & -51.012 & 0.127 & 48.583 \\
\hline 50 & 150 & 0.118 & 0.259 & -119.492 & 0.221 & -87.288 & 0.114 & 3.390 \\
\hline
\end{tabular}




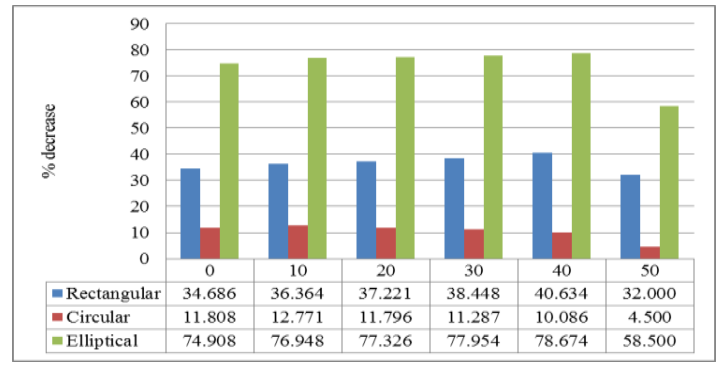

Chart -13: Storey drift in X-direction (wog)

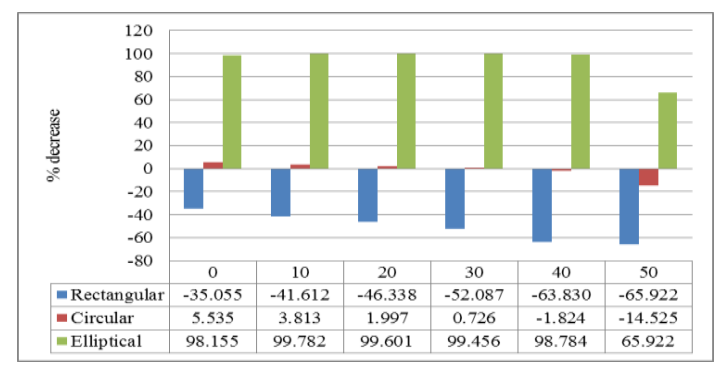

Chart -14: Storey drift in Y-direction (wog)

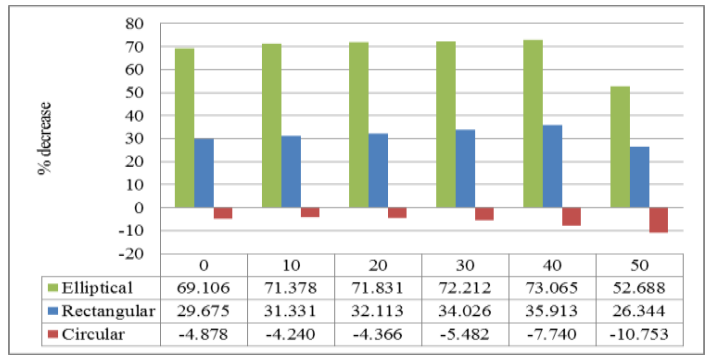

Chart -15: Storey drift in X-direction (wg)

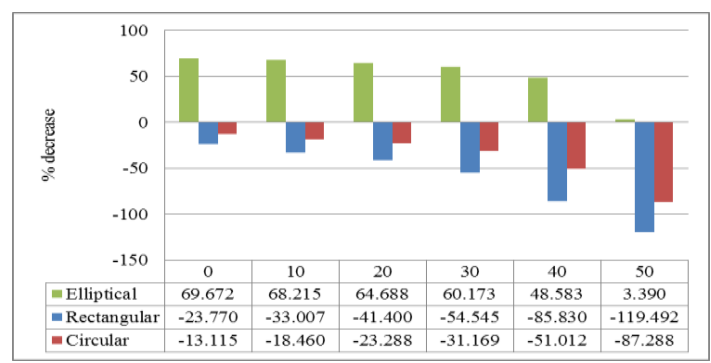

Chart -16: Storey drift in Y-direction (wg)

\subsection{Storey Shear}

The summation of design lateral forces at levels above the storey under consideration. The storey shear for square, rectangular, circular and elliptical shape building are

compared and it is graphically represented in chart $17,18,19,20$.

Table -19: Comparison of storey shear without gust factor (X-direction) $(\mathrm{kN})$

\begin{tabular}{|c|c|c|c|c|c|c|c|c|}
\hline \multirow{2}{*}{$\begin{array}{l}\text { Storey } \\
\text { No. }\end{array}$} & \multirow{2}{*}{$\begin{array}{l}\text { Height } \\
\text { m }\end{array}$} & Square & \multicolumn{2}{|c|}{ Rectangular } & \multicolumn{2}{|c|}{ Circular } & \multicolumn{2}{|c|}{ Elliptical } \\
\hline & & $\begin{array}{c}\text { Storey shear } \\
\qquad(\mathrm{kN})\end{array}$ & $\begin{array}{l}\text { Storey } \\
\text { shear } \\
(\mathrm{kN})\end{array}$ & $\begin{array}{c}\% \\
\text { Decrease }\end{array}$ & $\begin{array}{l}\text { Storey } \\
\text { shear } \\
(\mathrm{kN})\end{array}$ & $\begin{array}{c}\% \\
\text { Decrease }\end{array}$ & $\begin{array}{c}\text { Storey } \\
\text { shear } \\
(\mathrm{kN})\end{array}$ & $\begin{array}{c}\% \\
\text { Decrease }\end{array}$ \\
\hline 0 & 0 & 13980.735 & 9181.982 & 34.324 & 11393.930 & 18.503 & 3551.140 & 74.600 \\
\hline 10 & 30 & 12041.085 & 7931.808 & 34.127 & 9842.439 & 18.260 & 3067.626 & 74.524 \\
\hline 20 & 60 & 9401.61 & 6220.733 & 33.833 & 7718.960 & 17.897 & 2405.855 & 74.410 \\
\hline 30 & 90 & 6550.215 & 4333.736 & 33.838 & 5377.159 & 17.909 & 1676.105 & 74.411 \\
\hline 40 & 120 & 3485.775 & 2320.950 & 33.417 & 2879.251 & 17.400 & 897.645 & 74.248 \\
\hline 50 & 150 & 321.465 & 215.245 & 33.043 & 267.123 & 16.904 & 83.248 & 74.104 \\
\hline
\end{tabular}

Table -9: Comparison of storey shear without gust factor (Y-direction) $(\mathrm{kN})$

\begin{tabular}{|c|c|c|c|c|c|c|c|c|}
\hline \multirow{2}{*}{$\begin{array}{c}\text { Storey } \\
\text { No. }\end{array}$} & \multirow{2}{*}{$\begin{array}{l}\text { Height } \\
\mathrm{m}\end{array}$} & Square & \multicolumn{2}{|c|}{ Rectangular } & \multicolumn{2}{|c|}{ Circular } & \multicolumn{2}{|c|}{ Elliptical } \\
\hline & & $\begin{array}{c}\text { Storey } \\
\text { shear } \\
(\mathrm{kN})\end{array}$ & $\begin{array}{c}\text { Storey } \\
\text { shear } \\
(\mathrm{kN})\end{array}$ & $\begin{array}{c}\% \\
\text { Decrease }\end{array}$ & $\begin{array}{c}\text { Storey } \\
\text { shear } \\
(\mathrm{kN})\end{array}$ & $\begin{array}{c}\% \\
\text { Decrease }\end{array}$ & $\begin{array}{c}\text { Storey } \\
\text { shear } \\
(\mathrm{kN})\end{array}$ & $\begin{array}{c}\% \\
\text { Decrease }\end{array}$ \\
\hline 0 & 0 & 13975.845 & 17996.685 & -28.770 & 12092.5306 & 13.475 & 3555.0198 & 74.563 \\
\hline 10 & 30 & 12036.195 & 15546.344 & -29.163 & 10429.4282 & 13.349 & 3071.5056 & 74.481 \\
\hline 20 & 60 & 9396.72 & 12192.637 & -29.754 & 8179.7516 & 12.951 & 2409.7343 & 74.356 \\
\hline 30 & 90 & 6518.055 & 8494.122 & -30.317 & 5698.7774 & 12.569 & 1676.1052 & 74.285 \\
\hline 40 & 120 & 3453.615 & 4549.063 & -31.719 & 3051.5243 & 11.643 & 897.6452 & 74.009 \\
\hline 50 & 150 & 289.305 & 421.880 & -45.825 & 282.998 & 2.180 & 83.2475 & 71.225 \\
\hline
\end{tabular}


Table -9: Comparison of storey shear with gust factor (X-direction) $(\mathrm{kN})$

\begin{tabular}{|c|c|c|c|c|c|c|c|c|}
\hline \multirow{2}{*}{$\begin{array}{l}\text { Storey } \\
\text { No. }\end{array}$} & \multirow{2}{*}{$\begin{array}{c}\text { Height } \\
\text { m }\end{array}$} & \multirow{2}{*}{$\begin{array}{c}\text { Square } \\
\text { Storey } \\
\text { shear } \\
(\mathrm{kN})\end{array}$} & \multicolumn{2}{|c|}{ Rectangular } & \multicolumn{2}{|c|}{ Circular } & \multicolumn{2}{|c|}{ Elliptical } \\
\hline & & & $\begin{array}{c}\text { Storey } \\
\text { shear } \\
(\mathrm{kN}) \\
\end{array}$ & $\begin{array}{c}\% \\
\text { Decrease }\end{array}$ & $\begin{array}{c}\text { Storey } \\
\text { shear } \\
(\mathrm{kN}) \\
\end{array}$ & $\begin{array}{c}\% \\
\text { Decrease }\end{array}$ & $\begin{array}{l}\text { Storey } \\
\text { shear } \\
(\mathrm{kN}) \\
\end{array}$ & $\begin{array}{c}\% \\
\text { Decrease }\end{array}$ \\
\hline 0 & 0 & 12573.615 & 8915.481 & .094 & 12282.829 & 2.313 & 3969.000 & 68.434 \\
\hline 10 & 30 & 11068.875 & 7859.618 & 28.994 & 10806.057 & 2.374 & 3498.495 & 68.393 \\
\hline 20 & 60 & 8805.45 & 6265.258 & 28.848 & 8487.108 & 3.615 & 2783.305 & 68.391 \\
\hline 30 & 90 & 6210.885 & 4339.087 & 30.137 & 5967.661 & 3.916 & 1965.336 & 68.357 \\
\hline 40 & 120 & 3352.275 & 2338.503 & 30.241 & 3214.616 & 4.106 & 1058.619 & 68.421 \\
\hline 50 & 150 & 314.535 & 218.360 & 30.577 & 300.688 & 4.403 & 99.469 & 68.376 \\
\hline
\end{tabular}

Table -9: Comparison of wind loads with gust factor (Y-direction) (kN)

\begin{tabular}{|c|c|c|c|c|c|c|c|c|}
\hline \multirow{2}{*}{$\begin{array}{l}\text { Storey } \\
\text { No. }\end{array}$} & \multirow{2}{*}{$\begin{array}{l}\text { Height } \\
\mathrm{m}\end{array}$} & \multirow{2}{*}{$\begin{array}{c}\text { Square } \\
\text { Storey } \\
\text { shear } \\
(\mathrm{kN})\end{array}$} & \multicolumn{2}{|c|}{ Rectangular } & \multicolumn{2}{|c|}{ Circular } & \multicolumn{2}{|c|}{ Elliptical } \\
\hline & & & $\begin{array}{c}\text { Storey } \\
\text { shear } \\
(\mathrm{kN})\end{array}$ & $\begin{array}{c}\% \\
\text { Decrease }\end{array}$ & $\begin{array}{c}\text { Storey } \\
\text { shear } \\
(\mathrm{kN})\end{array}$ & $\begin{array}{c}\% \\
\text { Decrease }\end{array}$ & $\begin{array}{l}\text { Storey } \\
\text { shear } \\
(\mathrm{kN})\end{array}$ & $\begin{array}{c}\% \\
\text { Decrease }\end{array}$ \\
\hline 0 & 0 & 12587.025 & 14815.455 & -17.704 & 13013.246 & -3.386 & 3727.399 & 70.387 \\
\hline 10 & 30 & 11081.385 & 13002.693 & -17.338 & 11448.710 & -3.315 & 3272.592 & 70.468 \\
\hline 20 & 60 & 8817.96 & 10313.509 & -16.960 & 8991.669 & -1.970 & 2595.666 & 70.564 \\
\hline 30 & 90 & 6210.885 & 7257.578 & -16.853 & 6322.492 & -1.797 & 1824.255 & 70.628 \\
\hline 40 & 120 & 3352.275 & 3881.193 & -15.778 & 3405.835 & -1.598 & 982.173 & 70.701 \\
\hline 50 & 150 & 314.535 & 361.898 & -15.058 & 318.557 & -1.279 & 91.655 & 70.860 \\
\hline
\end{tabular}

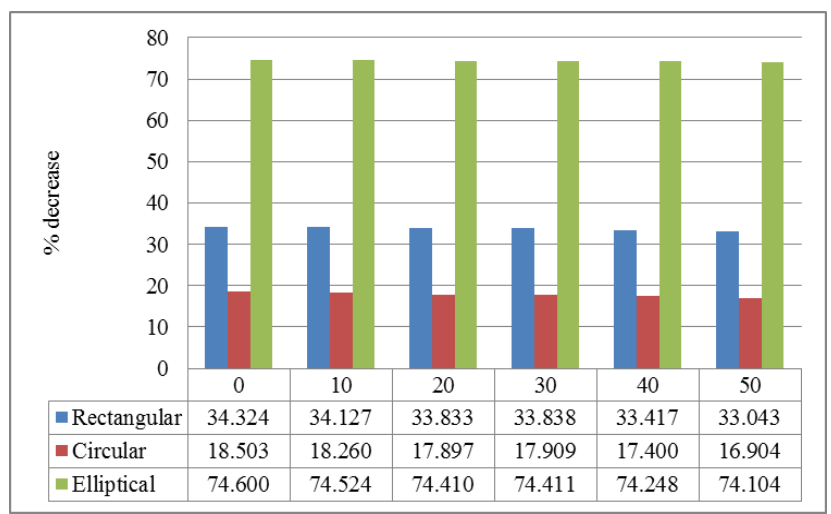

Chart- 17: Storey shear in X-direction (wog)

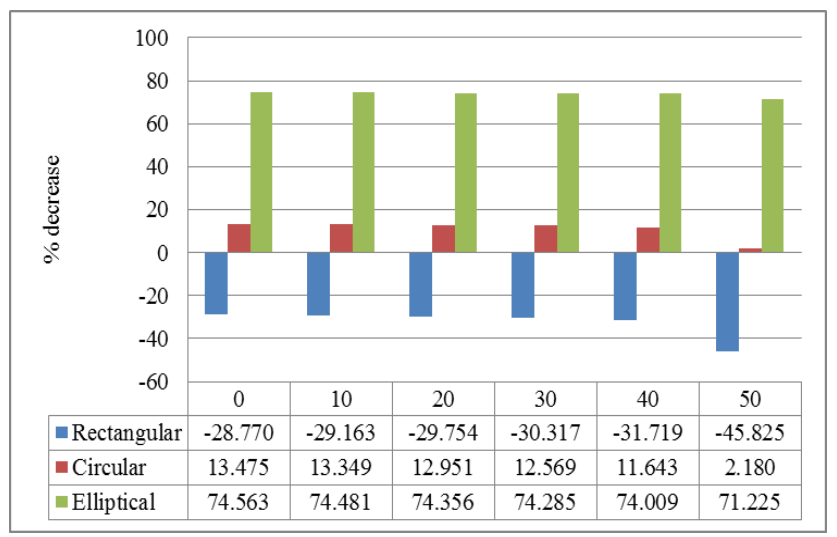

Chart- 18: Storey shear in Y-direction (wog)

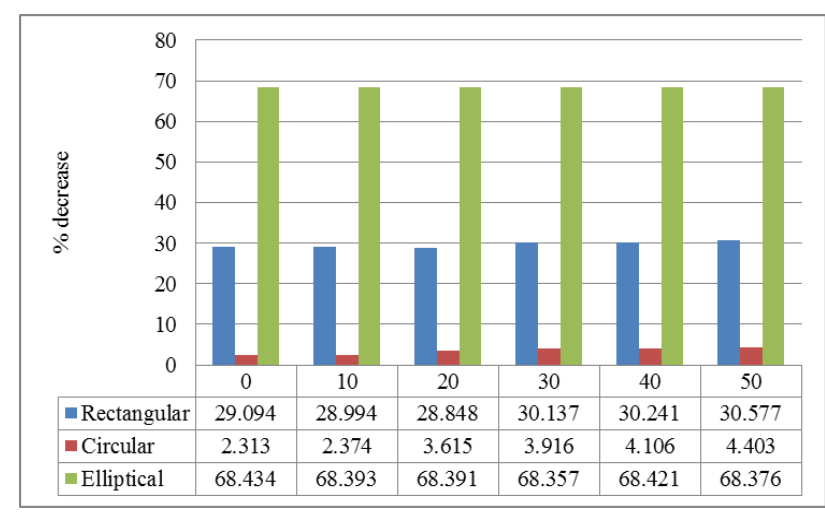

Chart- 19: Storey shear in X-direction (wg)

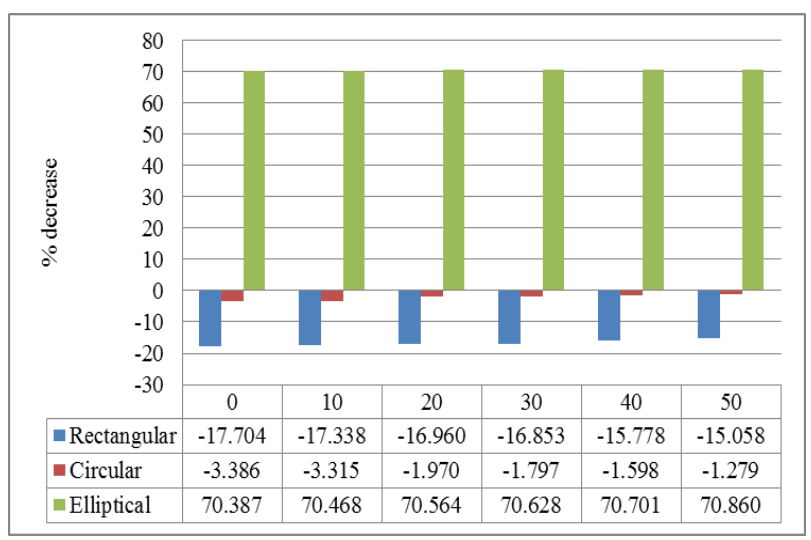

Chart- 20: Storey shear in Y-direction (wg) 


\section{CONCLUSION}

1. The percentage reduction in peak wind intensity for circular building is $16.96 \%, 74.115 \%$ for elliptical building and it is more by $31.183 \%$ for rectangular building when compared with square building without gust factor. The percentage reduction in peak wind intensity for circular building is $4.471 \%, 68.392 \%$ for elliptical building and it is more by $15 \%$ for rectangular building when compared with square building with gust factor.

2. The percentage reduction in peak displacement in circular building is $11.73 \%, 76.74 \%$ in elliptical building ,31.18\% in rectangular building without gust factor and $28.94 \%$ in rectangular building, $71.51 \%$ in elliptical building and it is more by $3.08 \%$ in circular building with gust factor in longitudinal direction when compared with square building. The percentage reduction in peak displacement in circular building is $5.41 \%, 71.76 \%$ for elliptical building and it is more by $42.67 \%$ for rectangular building without gust factor and it is $70.18 \%$ in elliptical building, it is more by 27 $\%$ in rectangular, $2.40 \%$ in circular building when compared with square building with gust factor in transverse direction.

3. The percentage reduction in peak drift in circular building is $4.50 \%, 58.50 \%$ in elliptical building , 32 $\%$ in rectangular building without gust factor and 26.44 $\%$ in rectangular building, $52.68 \%$ in elliptical building and it is more by $10.75 \%$ in circular building with gust factor in longitudinal direction when compared with square building. The percentage reduction in peak drift in elliptical building is $65.92 \%$ and it is more by 65.92 $\%$ for rectangular building and $14.52 \%$ in circular building without gust factor and it decreased by $3.39 \%$ in elliptical building, it is more by $119.49 \%$ in rectangular, $87.28 \%$ in circular building when compared with square building with gust factor in transverse direction.

4. The percentage reduction in peak storey shear in circular building is $16.90 \%, 74.10 \%$ in elliptical building, $33 \%$ in rectangular building without gust factor and $30.57 \%$ in rectangular building, $4.40 \%$ in elliptical building and $68.37 \%$ in circular building with gust factor in longitudinal direction when compared with square building. The percentage reduction in peak drift in circular building is 2.18 $\%, 71.22 \%$ in elliptical building and it is more by $45.85 \%$ for rectangular building without gust factor and it decreased by $70.86 \%$ in elliptical building, it is more by $14 \%$ in rectangular, $1.27 \%$ in circular building when compared with square building with gust factor in transverse direction.

5. The gust factor method uses the statistical concepts of a stationary time series to calculate the response of structure to a gusty wind. Hence it is important for the estimation of wind loads on the flexible structures.

6. Buildings having circular or elliptical plan forms have a smaller surface perpendicular to the wind direction, the wind pressure is less than in prismatic buildings.
7. From the above results, with the change in shape of building from square to elliptical the wind intensity, storey drifts, the lateral displacements, storey shear of the building decreased. Hence it is conclude that wind load is reduced by maximum percentage with an elliptical plan.

\section{REFERENCES}

[1] J. A. Amin and A. K. Ahuja "Experimental study of wind-induced pressures on buildings of various geometries" Journal of engineering, science and technology Vol. 3, No. 5, 2011, pp. 1-19.

[2] Prof. Sarita Singla, Taranjeet Kaur, Megha Kalra and Sanket Sharma "Behaviour of R.C.C. tall buildings having different shapes subjected to wind load" Journal on civil and environmental engineering 02, 2012, 3-17.

[3] P. Harikrishna, A. Abraham, S. Arunachalam, S. Selvi Rajan and G. Ramesh Babu "Pressure measurement studies on a model of a tall building with different plan shapes along the height" ,The seventh Asia-Pacific conference on wind engineering, Taipei, Taiwan, November 8-12, 2009.

[4] Hemil Chauhan, Manish Pomal, Gyayak Bhuta "A comparative study of wind forces on high-rise buildings as per IS 875(III)-1987 and proposed draft code (2011)" Global research analysis, ISSN no.22778160, Vol. 2, No. 5, May 2013.

[5] ETABS nonlinear Version 9.1.6, Extended 3D analysis of the building systems, Computer and Structures.

[6] IS: 875-1987(part 3) "Code of practices for design loads (other than earth quake) for buildings and structures". Bureau of Indian standards, New Delhi

[7] Explanatory handbook on "Indian Standard Code Practice for Design Loads" (other than earthquake) for buildings and structures part 3 wind loads [IS 875 (PART 3): 1987]", Bureau of Indian standards, New Delhi.

\section{BIOGRAPHIES}

1. M. R. Wakchaure, Professor, Civil Engineering Department, Amrutvahini College Of Engineering, Sangamner, Maharashtra, India.

2. Sayali J. Gawali, Post Graduate Student,Civil Engineering Department, Amrutvahini College Of Engineering, Sangamner, Maharashtra 